Int. J. Dev. Biol. 62: 363-368 (2018)

https://doi.org/10.1387/ijdb.170269dg

\title{
Regeneration: sooner rather than later
}

\author{
SUSAN V. BRYANT and DAVID M. GARDINER* \\ University of California Irvine, Irvine, CA, USA
}

\begin{abstract}
The explosive growth of information from genetics and genomics has led to an appreciation of the conservation of gene regulatory networks between organisms, and between development and regeneration. With ever increasing knowledge, it will be possible to intervene therapeutically to regulate these networks, which will lead to new therapies to induce regeneration. The question then becomes how to do this, rather then when to try. Our thesis is that the time is now, and that this feat can be achieved by combining the insights provided by developmental biologists with the technologies being developed by biomaterial engineers, to achieve the goal of engineering regeneration. We thus envision regenerative engineering as the next step toward achieving the goal of human regeneration. Among the most important discoveries about regeneration from studies of salamanders that regenerate exceptionally well, is that both pattern-following and pattern-forming cells are required. Much progress is being made toward understanding the former cells, but little is known about the cells that control positional information and pattern formation. Within the near future, it will become possible to provide the information needed for regeneration exogenously in the form of an engineered extracellular matrix that is a biomimetic of the endogenous information. Since growth factors (morphogens) can control pattern formation, an engineered grid could be based on spatially organized patterns of sulfation of glycosaminoglycans that control the behavior of cells by modulating morphogen activity. Progress in engineering the positional information grid for regeneration will necessitate learning the sulfation codes associated with successful regeneration in animals such as salamanders.
\end{abstract}

KEY WORDS: regeneration, dedifferentiation, wound healing, blastema, ECM

\section{Introduction}

Experimental biology has it roots in the quest to discover the principles and processes of embryonic and regenerative development. This all began with Lazzaro Spallanzani in the $18^{\text {th }}$ Century who demonstrated that embryonic development was initiated by fertilization of the ovum by sperm, and also discovered regenerative development of an amputated newt limb (Dinsmore, 1992; Morgan, 1901). Since that time, researchers have had the thought in the back of their minds that someday it might be possible to discover how to unlock the same intrinsic regenerative abilities in humans. With each advance in technology (e.g. electron microscopy or monoclonal antibodies), researchers have been excited that perhaps it was the time to discover how regeneration works. Discoveries in developmental genetics over the past couple of decades, combined with advances in genomics and gene-editing tools have once again brought us to the point of considering the possibility of opening the lock to human regeneration.

As authors of this essay, we offer our perspective of how the field of regeneration biology has advanced over this most recent period of history, and address the major challenges to achieving the goal of human regeneration. Our assignment is neither to provide a broad overview of regeneration, nor to catalog the ever-growing list of genes expressed during regeneration; rather the goal is to provide a more personal perspective based on the many years of contributions from our lab to regeneration studies. We note that many established labs, as well as those of new investigators are generating exciting new insights into the cellular and molecular mechanisms of regeneration. Our focus on work from our own lab is not intended to ignore or minimize these advances toward enhanced regenerative therapies. In addition, we view regeneration as a basic and conserved biological property, and thus discoveries from all regeneration models, whether vertebrate or invertebrate, are important in achieving this goal. Since our research model has been the urodele amphibian (newt in the beginning, and then

Abbreviations used in this paper: ECM, extracellular matrix.

\footnotetext{
*Address correspondence to: David M. Gardiner. Department of Developmental and Cell Biology, University of California Irvine, Irvine, CA 92697 USA. Tel: +1-(949) 824-2792. E-mail: dmgardin@ @ci.edu - web: regeneration.bio.uci.edu - iD https://orcid.org/0000-0001-9003-7496
} 
the axolotl in recent decades), this essay focuses on our thoughts that have emerged from our work with this model organism while acknowledging the importance of contributions and insights from a wide range of model organisms for regeneration.

For one of us (SVB), regeneration research started with the concept of positional information formulated by the iconic Lewis Wolpert. The combination of his dramatic personality and his thinking about science in a different way captivated Sue, a young undergraduate at King's College at the time. Along the way, the other of us (DMG) was in turn captivated by the ideas of the Polar Coordinate Model that formalized how positional information integrates growth and pattern formation during regeneration (Bryant et al., 1981; Bryant, Gardiner, 2016; French et al., 1976). Collectively we have spent nine decades thinking about how regeneration works, and although there will always be more to learn, we like others before us, argue that we have progressed to the point where it is time to focus on how to make regeneration work for humans. There always will be more details to discover, but it is important to remember that people today are in need of enhanced regenerative therapies. Thus more research is good, but the urgency of those in need today should drive efforts to make regeneration happen sooner rather than later.

Our optimism for success in achieving human regeneration is based mainly on the explosive growth of information from genetics and genomics of the past several years that have identified the key signaling networks that control development. Most importantly, there is now an appreciation of the conservation of these networks between organisms, and between development and regeneration. Thus the diversity of biological processes between animals is not a consequence of differences in these networks, but of differences in regulation of these conserved networks in time and space. Thus we will continue to learn ever more about the details of these networks, in particular how non-protein coding regions of the genome regulate these networks. With ever increasing knowledge, it is becoming possible to intervene therapeutically to regulate these networks, which will lead at some point to new therapies to induce regeneration. The question then becomes how to do this rather than when to try. If we can succeed, then history will show that the time was now.

Although the ultimate goal is to unlock the intrinsic regenerative abilities of humans (assuming they exist as discussed below), this may take a very long time, and we need to remember the urgency. To achieve the goal of making the most of what we know now, we presume that regeneration is a conserved biological process that occurs to a greater or lesser extent in all animals, and that some animals do it better (e.g. salamanders) than others (e.g. humans). Therefore, studying how regeneration works in the good-regenerators will identify the gene regulatory networks (GRNs) that need to be targeted therapeutically in the not-so-good regenerators. Targeting these conserved pathways will necessitate delivering activating and inhibiting signals to the appropriate target cells at the right time and place. This feat can be achieved by combining the insights provided by developmental biologists with the technologies being developed by biomaterial engineers to achieve the goal of engineering regeneration. We thus envision regenerative engineering as the next step toward achieving the goal of human regeneration.

\section{What we have learned about how regeneration works}

A focus of modern biology is on the ever-increasing details of developmental processes. Although this approach is designed to identify the nature of the system in an unbiased fashion, there also is value in stepping back to survey the landscape and identify the broader organizing principles and concepts of the system being studied, e.g. regeneration. From our perspective, one of the most important points to remember is that if an animal has the ability to make a structure (e.g. a limb) during embryonic development, then it also has the ability to remake it during regenerative development (i.e. the requisite genes and GRNs are present). Although there may be multiple ways to regenerate a structure, one obvious way is to do again what was done before. If regenerative failure in humans is not about a limitation in our genetics, then why do we not regenerate as well as a salamander? As noted above, it is becoming increasing evident that the biological outcome is dictated by how conserved genes and GRNs are controlled by non-protein coding regions of the genome, such as tissue regeneration enhancer elements (TREEs) that trigger gene expression at injury sites (Kang et al., 2016). Although research in the area is relatively

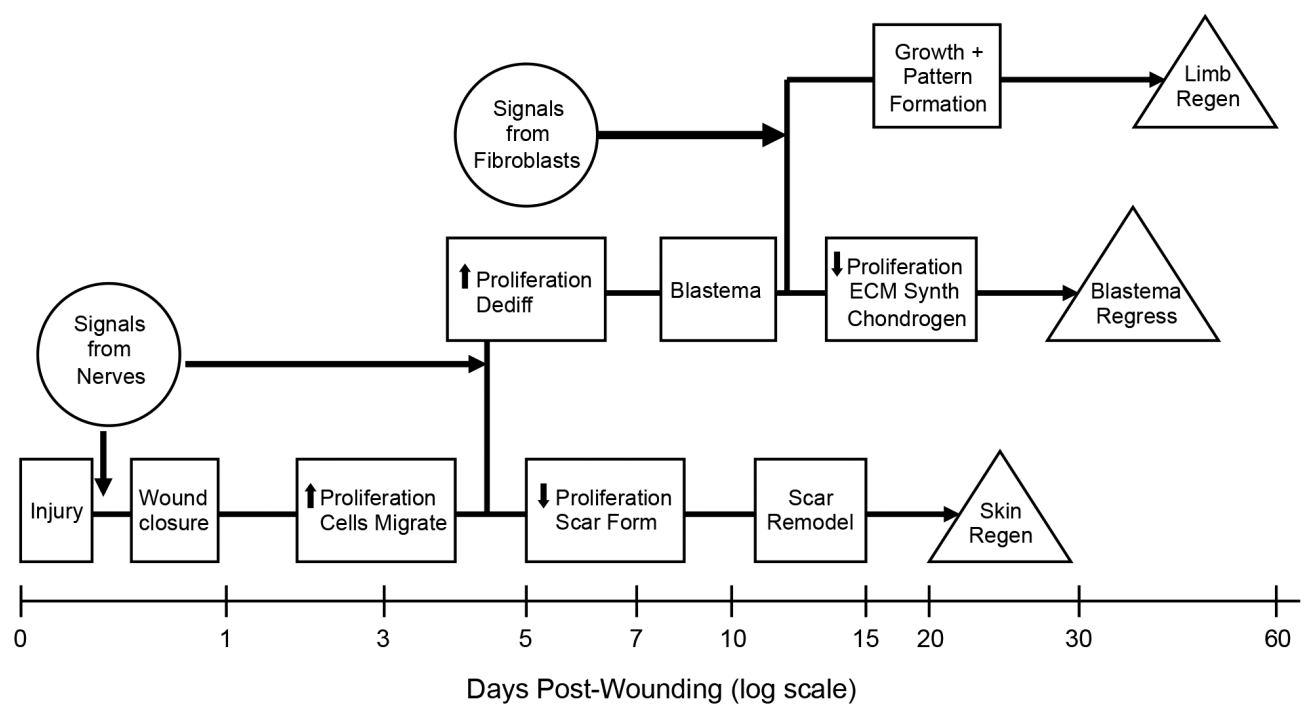

Fig. 1. Regeneration is a step-wise process. In response to injury, a skin wound in an axolotl either regenerates the skin, or forms a blastema that may or not progress to form a new limb. The different outcomes are a consequence of proregenerative signals associated with injury, the presence of nerves, and cells with positional information. Signals from the nerve function early and at later times to recruit connective tissue cells that dedifferentiate and proliferate to form the blastema. If no further signals are provided, the induced blastema eventually stops growing and is integrated into the host tissues. If additional signals are provided by fibroblasts with positional information in a skin graft (or ECM graft) from the side of the limb opposite to the wound site, the ectopic blastema continues to grow and forms an ectopic limb. 
new, it seems reasonable to anticipate that many such TREEs will be identified in the near future. These discoveries likely will lead to important clinical advances in the field since they provide the opportunity for the regenerative engineering approach we envision as the way forward.

Key to this idea that regeneration occurs as a result of the orchestrated control of GRNs is a second principle, which is that regeneration is a stepwise process that is initiated in response to injury signals (Fig. 1). The outcome at each step will be determined by the presence or absence of signals that activate or inhibit critical GRNs. Much like Waddington's epigenetic landscape (see Noble, 2015), at each step the cells diverge along different pathways that may or not lead to the next critical step along the trajectory leading to regeneration (Endo et al., 2004). Consequently, a failure to progress along the regeneration pathway at any of the earlier steps will lead to regenerative failure (Muller et al., 1999). Thus the answer to the often-asked question as to why humans fail to regenerate is not that we lack special regeneration genes, but rather that the steps necessary for regeneration fail to lead to blastema formation and subsequent regeneration.

This brings us to a third principle of regeneration, which is that the road to regeneration leads thru blastema formation. Although some invertebrates reform body parts without first making a blastema (a process referred to classically as morphallaxis), the replacement of lost structures typically involves blastema formation (referred to epimorphic regeneration). The blastema is a transient developmental structure that is formed by the recruitment of cells from the stump tissues after injury, and that grows and eventually forms the missing structures (see McCusker et al., 2015)). In this regard, it is equivalent to embryonic development, and in the case of limb regeneration the blastema is equivalent to the limb bud (Muneoka, Bryant, 1982). Although they start with different cells (the limb bud from undifferentiated embryonic cells of the lateral plate mesoderm, and the blastema from stem cells and differentiated cells of the limb), at some point, they converge in terms of the behavior and fate of the cells (Bryant et al., 2002). This convergence has been demonstrated directly in the axolotl by reciprocal grafting between developing limb buds and regenerating limb blastemas. Each behaves the same as the other with regards to growth and pattern formation, which directly demonstrates the conservation of mechanisms between limb development and limb regeneration (Muneoka, Bryant, 1982; Muneoka, Bryant, 1984).

The blastema is more than a homogenous mass of undifferentiated cells. Although all tissues of the stump appear to contribute cells to the blastema, their relative contributions and fates are vastly different. Some cell types such as muscle and cartilage are underrepresented in the blastema relative to their abundance in the uninjured limb. In contrast, cells derived from the connective tissues are overrepresented such that nearly $50 \%$ the early blastema cells are of dermal origin even though connective tissue cells represent only about $20 \%$ of the cells of the uninjured limb (Muneoka et al., 1986). The relative abundance of blastema cells derived from different tissues thus changes during blastema formation and growth such that at the end of regeneration the original abundance of the different differentiated cell types is restored.

In addition to differences in contribution, cells that make up a blastema also differ in terms of their developmental potential. Some are lineage-restricted such as epidermal cells, muscle cells, and Schwann cells (Kragl et al., 2009). In contrast, the connective tissue lineage is diverse in terms of differentiated cells types, and thus cells within the connective tissue of the dermis are capable of giving rise to an array of tissues, such as cartilage, bone, loose connective tissue, ligaments, tendons and muscle fascia (Holder, 1989; Lheureux, 1983; McCusker et al., 2016; McCusker, Gardiner, 2013). In fact, progeny of dermal cells can regenerate the entire limb without contributions from other cells of the limb stump (Lheureux, 1983). In these studies, regeneration was inhibited by $\mathrm{x}$-irradiation, but could be rescued by a graft of unirradiated limb skin. The migration of lineage-restricted myoprogenitor cells in the stump was inhibited, and thus these regenerated limbs were muscle-less; however, other lineage-restricted cells such as blood vessels, and nerves along with associated Schwann cells migrated into to the regenerated limb and contributed to formation of the normal pattern. Thus some cells required to remake the lost structures are derived from well-characterized stem cell progenitors with restricted developmental potential, and their progeny migrate into the blastema after it has formed. The biology of these adult stem cells is the focus of considerable research, and we presume that with time, the challenge of controlling the behavior of these cells in order to engineer regeneration will be met. In contrast, very little is known about the identity and behavior of the connective tissue cells during regeneration, in spite of the evidence indicating their critical role in controlling regeneration in very good regenerators such as salamanders.

Although this dynamic and transient nature of the regeneration blastema is intuitively obvious, historically researchers have tended to refer to the blastema as a specific entity like other parts of the adult body. This becomes a problem when homogenizing the entire blastema to isolate proteins or mRNA, because it results in the loss of the spatial heterogeneity that is essential for achieving the outcome of sequentially rebuilding the lost body part. Although it will be challenging to engineer the spatial complexity to make a functional blastema, the good news is that it is most likely that we will just need to get the starting conditions right and then let the blastema develop from there. At later stages, an ectopically grafted blastema (e.g. to a wound on the side of the arm) will develop autonomously and form an ectopic limb (McCusker, Gardiner, 2013; Stocum, 1968). Similarly an ectopic blastema that is induced to form on the side of the arm will develop into an ectopic limb if cells with the appropriate positional information are recruited (Endo et al., 2004) (discussed below). Finally, denervation of a limb at early stages of blastema formation results in reintegration of the blastema cells into the stump and regenerative failure; whereas, once the blastema reaches the late bud stage, it will continue to develop and form a new limb without a nerve supply (see McCusker et al., 2015; Wallace, 1981). Thus it appears that the challenge will be to engineer a blastema that can progress to the transition between the medium and late bud stage of development. After that, the blastema is expected to function as a self-organizing system that will make a new limb without further input.

This difference in the behavior of cells of the early bud versus late bud blastema brings us to a fourth principle of regeneration. Tissue grafting studies to induce formation of supernumerary limb structures have demonstrated that there are two populations of cells involved in blastema formation, and that both are required for successful regeneration. As noted, there are the lineage-restricted progenitor cells (adult stem cells) that are required to make many of the structures of the limb (e.g. muscle, nerves, blood vessels 
and the epidermis). In addition, there are the cells that control the behavior of these progenitor cells in terms of where to go and wen to differentiate (i.e. they control formation of the new limb pattern) (McCusker et al., 2015). These pattern-forming cells are characterized by their ability to induce supernumerary limb pattern as predicted and formalized many years ago in the Polar Coordinate Model (Bryant et al., 1981; French et al., 1976). Grafting of cells into a site with different positional information induces growth and the creation of the information that was missing between the cells of the graft and the host site. From this we infer that these cells have positional information and are pattern-forming cells. In contrast, if no supernumerary structures are induced to form, the cells of the host and graft either have the same information, or are lacking information (i.e. are pattern-following cells) (McCusker et al., 2015).

In addition to the necessity of having cells with positional information, it also must be the case that the information of these cells can be dynamically regulated. Regeneration is by definition the making of new structures from old structures, and therefore the cells with old positional information must be able to give rise to progeny with the new, missing information. This reprogramming of positional information is associated with the process that classi-

\section{Grid Cells in Intact Limb}

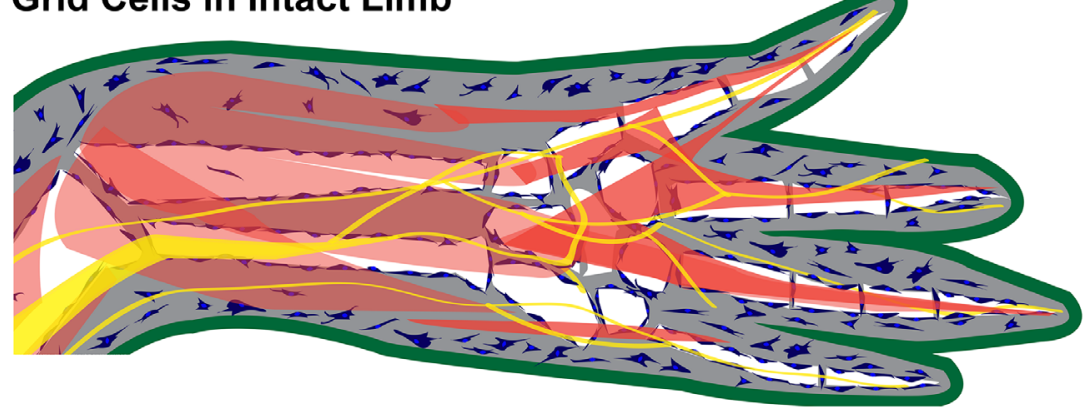

\section{Blastema}

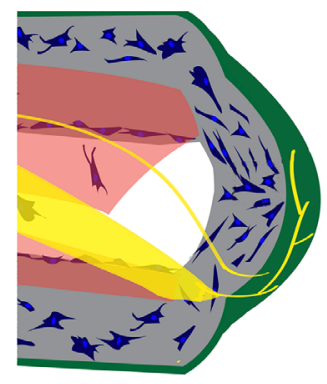

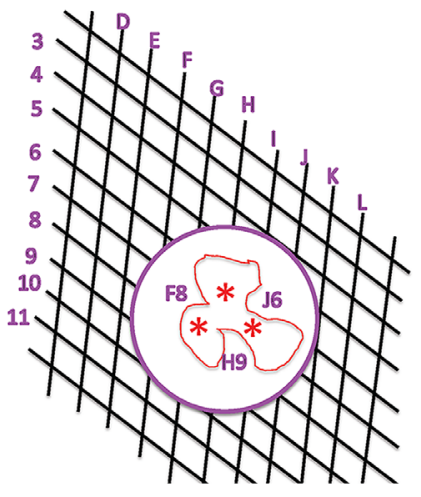

Fig. 2. Positional information grid cells control pattern formation. The positional information grid is composed of cells (blue) that are arrayed in a two-dimensional sheet throughout the loose connective tissues under the epidermis (green) and around internal structures (muscles - brown, bones - white, nerves - yellow, and blood vessels - red). When the limb is amputated, the blastema forms by the recruitment of grid cells in response to signaling from nerves (yellow) that interact with the thickened apical wound epithelium (green). Amputation is equivalent to creating a hole in the grid within which cells have information about their position along the proximal-distal $(D-L)$ and around the circumferential (3-11) limb axes. When the edges of the hole come together during wound healing, cells with different positional information come together and interact (red asterisks. These interactions stimulate growth and pattern formation (intercalation) resulting in repair of the grid. Modified with permission from (McCusker et al., 2015) and (Bryant, Gardiner, 2016). cally is referred to as dedifferentiation. The operational definition of dedifferentiation is the reversal of the differentiated state as can be viewed as being equivalent to endogenous reprogramming comparable to induced pluripotent stem cells (iPSCs), resulting (McCusker, Gardiner, 2013; decusker, Gardiner, 2014), and the acquisition of increased (Han et al., 2005; McCusker et al., 2015; McCusker, Gardiner, that corresponds to the host site when grafted. In contrast, in to redifferentiate at later stages of regeneration, pernumerary pattern when grafted (Iten, Bryant, 1975; McCusker, Gardiner, 2013). Similarly, treatment with retinoic acid (RA) can reprogram the PI of undifferentiated, PI-labile blastema cells, but McCusker et al., 2014).

Little is known about the biology of the patternforming cells, other than that they reside in connective tissues. Similarly, little is known about the chemical basis of the positional information that is made by and responded to by the pattern-forming cells. The PCM predicts that positional information is distributed as a two-dimensional grid that underlies the epidermis and wraps around all the internal tissues (Fig. 2) (Bryant et al., 1981; French et al., 1976; McCusker et al., 2015). Thus the pattern-forming cells are distributed throughout the loose connective tissues of the body where they encode a positional address along the proximal-distal and circumferential axes, much like a postal zip code. Research in recent years has led to an appreciation of how macromolecules in the extracellular matrix (ECM) function to mediate signaling between cells, and it is likely that positional information is a property of the ECM. Direct evidence of this function of the ECM during regeneration comes from studies in the axolotl involving the grafting of decellularized ECM from both axolotl and mouse tissues (Phan et al., 2015). Among the many biologically active macromolecules in the ECM, sulfated glycosaminoglycans (e.g. heparan sulfate proteoglycan, HSPG) are likely candidates for regulators of positional information. HSPGs control the activity of a number of growth factors/morphogens, and specific patterns of sulfation can differentially regulate the activity of specific morphogens (Sarrazin et al., 2011; Yayon et al., 1991). HSPG-dependent pattern-inducing activity of both axolotl and mouse ECM differs between anterior and posterior limb tissues, which is a property that is expected to be associated with positional information. The enzymes that synthesize these sulfation patterns are expressed in spatial and temporal domains that are predicted to result in regional differences in the ECM that would differentially regulate FGF signaling (Phan et al., 2015; Sarrazin et al., 2011; Yayon et al., 1991). 


\section{What can we do next?}

There always will be more to learn about regeneration, and going forward the goal should be to focus our research efforts to build knowledge that can be applied to improve the outcome of regenerative therapies. Among those efforts it will be important to use genetics and genomics to tease apart the regulatory codes for controlling proregenerative GRNs, for example the discovery of TREEs discussed above (Kang et al., 2016). The goal is not to edit the human genome, which likely will not be necessary given the conservation of signals and pathways between animals. Rather we want to take advantage of nature's free lessons provided by animals such as salamanders that are very good at regeneration, to discover how cells can response to signals from other cells and the ECM so as to move stepwise along the regeneration pathway. Knowing the GRNs involved in this process will enable us to use the tools of regenerative engineering to guide human cells as necessary.

Most ongoing studies of the cells involved in regeneration are focused on the pattern-following cells, with the goal of expanding and differentiating them in the right place to rebuild the missing body parts. Many of these are well characterized in terms of their embryonic development, and there are validated markers for the pathways leading to their differentiation (e.g. neurons derived from the neural plate and myoprogenitor cells derived from lateral plate mesoderm). Given the importance of the pattern-forming cells in regeneration, we find it puzzling that so little progress has been made in discovering and validating marker genes for these cells (broadly referred to as fibroblasts). The paucity of markers for subpopulations of fibroblasts is a major barrier to studying their behaviors in response to injury. In the end, our ability to induce regeneration in humans will be dependent on being able to regulate the behavior of these cells. Progress toward this end has been achieved through the discovery of mammalian signaling molecules associated with nerves (a cocktail of FGFs and BMPs) that can recruit these cells to the wound site leading to dedifferentiation and blastema formation (Makanae et al., 2013; Makanae et al., 2016).

Achieving the goal of endogenous regeneration may be a long time away in the future, and will require an understanding how positional information is encoded and how it is regenerated. In salamanders, this occurs as a consequence of the pattern-forming cells communicating with each other and with the ECM. Over the shorter term, we envision that the positional information needed for regeneration can be provided exogenously in the form of an engineered ECM that is a biomimetic of the endogenous information grid. This engineered grid would be based on spatially organized patterns of specific HSPG modifications that would control the behavior of cells by modulating morphogen activity (e.g. FGFs). Progress in engineering the positional information grid for regeneration will necessitate learning the HSPG sulfation codes associated with successful regeneration in animals such as salamanders.

\section{Acknowledgements}

We thank former members of our lab, especially Drs. Ken Muneoka, Tetsuya Endo, Akira Satoh, Anne Phan, and Catherine McCusker for helping develop our views of how regeneration works. Thanks also go to Dr. Cato Laurencin for his insights into the convergence of regeneration biology and engineering leading to the emergence of regenerative engineering for inducing human regeneration.

\section{References}

BRYANT SV, ENDO T, GARDINER DM (2002). Vertebrate limb regeneration and the origin of limb stem cells. Int J Dev Biol 46: 887-896.

BRYANT SV, FRENCH V, BRYANT PJ (1981). Distal regeneration and symmetry. Science 212: 993-1002.

BRYANT SV, GARDINER DM (2016). The relationship between growth and pattern formation. Regeneration (Oxf) 3: 103-122.

DINSMORE CE (1992). The foundations of contemporary regeneration research: historical perspectives. Monogr Dev Biol. 23: 1-27.

ENDO T, BRYANT SV, GARDINER DM (2004). A stepwise model system for limb regeneration. Dev Biol 270: 135-145.

FRENCH V, BRYANTPJ, BRYANT SV (1976). Pattern regulation in epimorphic fields. Science 193: 969-981.

HAN M, YANG X, TAYLOR G, BURDSAL CA, ANDERSON RA, MUNEOKAK (2005) Limb regeneration in higher vertebrates: developing a roadmap. Anat Rec $B N e w$ Anat 287: 14-24.

HOLDER N (1989). Organization of connective tissue patterns by dermal fibroblasts in the regenerating axolotl limb. Development 105: 585-593.

ITEN LE, BRYANT SV (1975). The interaction between the blastema and stump in the establishment of the anterior--posterior and proximal--distal organization of the limb regenerate. Dev Biol 44: 119-147.

KANG J, HUJ, KARRAR, DICKSONAL, TORNINI VA, NACHTRABG, GEMBERLING M, GOLDMAN JA, BLACK BL, POSS KD (2016). Modulation of tissue repair by regeneration enhancer elements. Nature 532: 201-206.

KRAGL M, KNAPP D, NACU E, KHATTAK S, MADEN M, EPPERLEIN HH, TANAKA EM (2009). Cells keep a memory of their tissue origin during axolotl limb regeneration. Nature 460: 60-65.

LHEUREUX E (1983). The origin of tissues in the $x$-irradiated regeneratin limb of the newt Pleurodeles waltilii. In Limb Development and Regeneration, Part A (Eds JF Fallon and Al Caplan). Alan R. Liss, Inc., New York, pp. 455-465.

MADEN M (1983). The effect of vitamin A on the regenerating axolotl limb. J Embryol Exp Morphol 77: 273-295.

MADEN M (1982). Vitamin A and pattern formation in the regenerating limb. Nature 295: 672-675.

MAKANAE A, HIRATA A, HONJO Y, MITOGAWA K, SATOH A (2013). Nerve independent limb induction in axolotls. Dev Biol 381: 213-226.

MAKANAE A, MITOGAWA K, SATOH A (2016). Cooperative inputs of Bmp and Fg signaling induce tail regeneration in urodele amphibians. Dev Biol 410: 45-55.

MCCUSKER C, BRYANT SV, GARDINER DM (2015). The axolotl limb blastema: cellular and molecular mechanisms driving blastema formation and limb regeneration in tetrapods. Regeneration 2: 54-71.

MCCUSKER C, LEHRBERG J, GARDINER D (2014). Position-specific induction of ectopic limbs in non-regenerating blastemas on axolotl forelimbs. Regeneration 1: 27-34.

MCCUSKER CD, DIAZ-CASTILLO C, SOSNIKJ, Q PHANA, GARDINERDM (2016). Cartilage and bone cells do not participate in skeletal regeneration in Ambystoma mexicanum limbs. Dev Biol 416: 26-33.

MCCUSKER CD, GARDINER DM (2013). Positional information is reprogrammed in blastema cells of the regenerating limb of the axolotl (Ambystoma mexicanum). PLOS ONE 8: e77064.

MCCUSKER CD, GARDINERDM (2014). Understanding positional cues in salamander limb regeneration: implications for optimizing cell-based regenerative therapies. Dis Model Mech 7: 593-599.

MORGAN TH (1901). Regeneration. The Macmillan Company, New York.

MULLERTL, NGO-MULLER V, REGINELLIA, TAYLOR G, ANDERSONR, MUNEOKA $K$ (1999). Regeneration in higher vertebrates: limb buds and digit tips. Semin Cell Dev Biol 10: 405-413.

MUNEOKA K, BRYANT SV (1984). Cellular contribution to supernumerary limbs in the axolotl, Ambystoma mexicanum. Dev Biol 105: 166-178.

MUNEOKAK, BRYANT SV (1982). Evidence that patterning mechanisms in developing and regenerating limbs are the same. Nature 298: 369-371.

MUNEOKA K, FOX W, BRYANT SV (1986). Cellular contribution from dermis and 


\section{S.V. Bryant and D.M. Gardiner}

cartilage to the regenerating limb blastema in axolotls. Dev. Biol. 116: 256-260. NOBLE D (2015). Conrad Waddington and the origin of epigenetics. The Company of Biologists Ltd.

PHAN AQ, LEE J, OEI M, FLATH C, HWE C, MARIANO R, VU T, SHU C, MUNEOKA K, BRYANT SV, GARDINER DM (2015). Heparan Sulfates Mediate Positional Information by Position-Specific Growth Factor Regulation during Axolotl (Ambystoma mexicanum) Limb Regeneration. Regeneration 2: 182-201.

SARRAZIN S, LAMANNA WC, ESKO JD (2011). Heparan sulfate proteoglycans. Cold Spring Harb Perspect Biol 3: a004952-a004952.
STOCUM DL (1968). The urodele limb regeneration blastema: a self-organizing system. i. Differentiation in vitro. Dev Biol 18: 441-456.

WALLACE H (1981). Vertebrate Limb Regeneration. John Wiley and Sons, Chichester.

YAYON A, KLAGSBRUN M, ESKO JD, LEDER P, ORNITZ DM (1991). Cell surface, heparin-like molecules are required for binding of basic fibroblast growth factor to its high affinity receptor. Cell $64: 841-848$. 


\section{Further Related Reading, published previously in the Int. J. Dev. Biol.}

The head organizer in Hydra

Hans R. Bode

Int. J. Dev. Biol. (2012) 56: 473-478

https://doi.org/10.1387/ijdb.113448hb

Regeneration and pattern formation - an interview with Susan Bryant

Michael K. Richardson and Cheng Ming Chuong

Int. J. Dev. Biol. (2009) 53: 827-833

https://doi.org/10.1387/ijdb.082596mr

Vertebrate limb regeneration and the origin of limb stem cells

Susan V Bryant, Tetsuya Endo and David M Gardiner

Int. J. Dev. Biol. (2002) 46: 887-896

http://www.intjdevbiol.com/web/paper/12455626

Retinoic acid and limb regeneration--a personal view

Malcolm Maden

Int. J. Dev. Biol. (2002) 46: 883-886

http://www.intjdevbiol.com/web/paper/12455625

Positional information in vertebrate limb development; an interview with Lewis Wolpert Cheryll Tickle

Int. J. Dev. Biol. (2002) 46: 863-867

http://www.intjdevbiol.com/web/paper/12455621

5 yr ISI Impact Factor $(2016)=2.421$

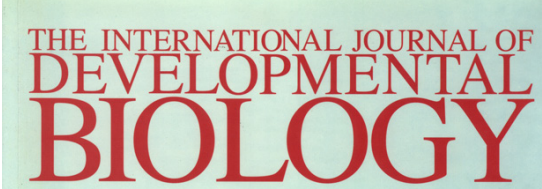

Volume 40 No. 4

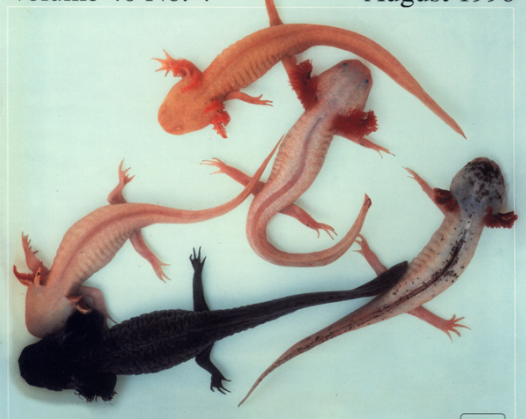

Developmental Biology of Urodeles
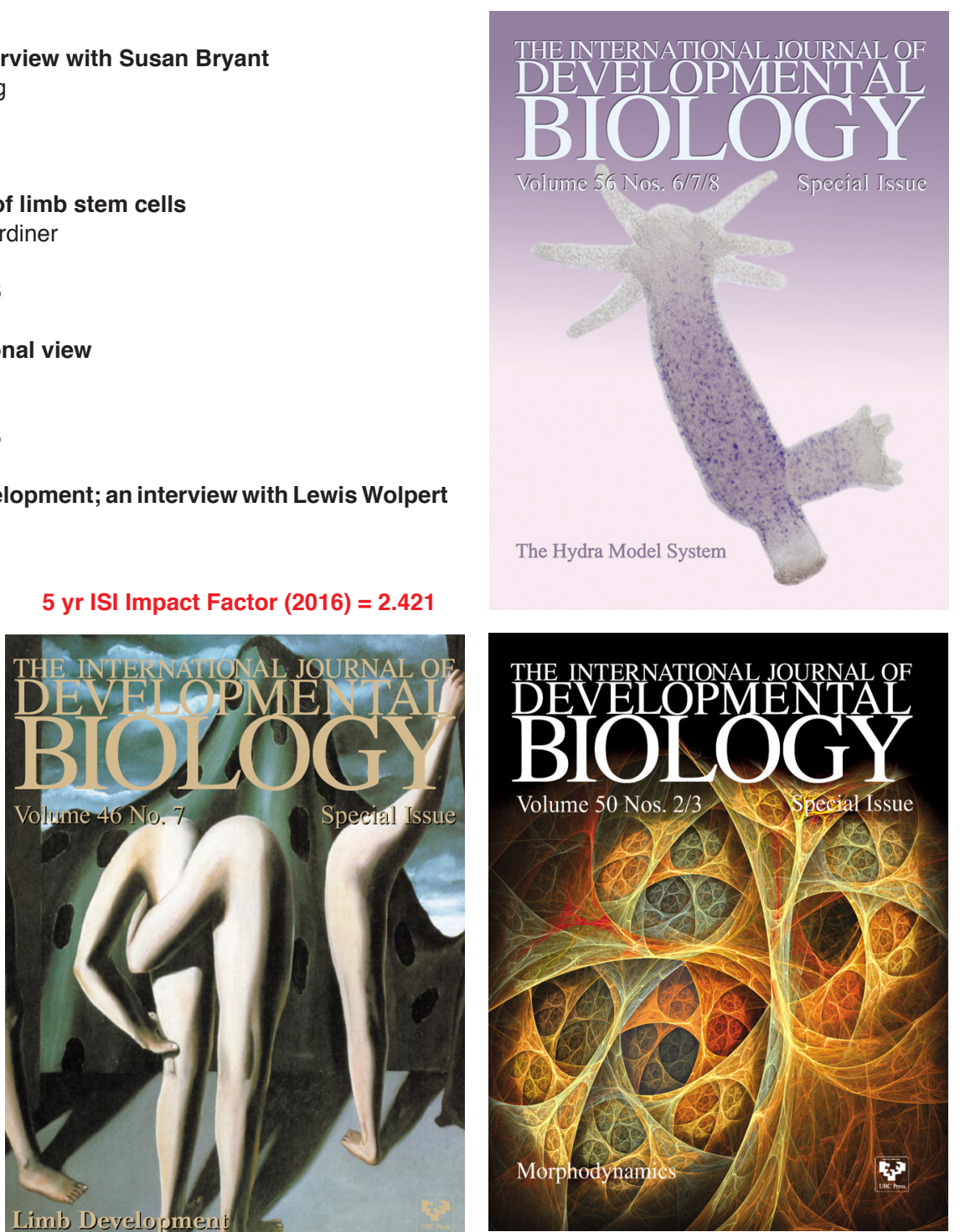